\title{
An optical trap for relativistic plasma ${ }^{a)}$
}

\author{
Ping Zhang, ${ }^{\text {b) }}$ Ned Saleh, Shouyuan Chen, Zhengming Sheng, ${ }^{\mathrm{c})}$ and Donald Umstadter \\ FOCUS Center, University of Michigan, Ann Arbor, Michigan 48109-2099
}

(Received 13 November 2002; accepted 14 February 2003)

\begin{abstract}
The first optical trap capable of confining relativistic electrons, with kinetic energy $\leqslant 350 \mathrm{keV}$ was created by the interference of spatially and temporally overlapping terawatt power, 400 fs duration laser pulses $\left(\leqslant 2.4 \times 10^{18} \mathrm{~W} / \mathrm{cm}^{2}\right)$ in plasma. Analysis and computer simulation predicted that the plasma density was greatly modulated, reaching a peak density up to 10 times the background density $\left(n_{e} / n_{0} \sim 10\right)$ at the interference minima. Associated with this charge displacement, a direct-current electrostatic field of strength of $\sim 2 \times 10^{11} \mathrm{eV} / \mathrm{m}$ was excited. These predictions were confirmed experimentally by Thomson and Raman scattering diagnostics. Also confirmed were predictions that the electron density grating acted as a multi-layer mirror to transfer energy between the crossed laser beams, resulting in the power of the weaker laser beam being nearly $50 \%$ increased. Furthermore, it was predicted that the optical trap acted to heat electrons, increasing their temperature by two orders of magnitude. The experimental results showed that the number of high energy electrons accelerated along the direction of one of the laser beams was enhanced by a factor of 3 and electron temperature was increased $\sim 100 \mathrm{keV}$ as compared with single-beam illumination.

(C) 2003 American Institute of Physics. [DOI: 10.1063/1.1566033]
\end{abstract}

\section{INTRODUCTION}

Trapping has often been used with great success to confine ultracold matter, leading to many important applications, such as Bose-Einstein condensation and matter-wave lasers. Traps capable of confining ultrahot matter, or plasma, have also been built for applications in the basic plasma research and thermonuclear fusion. For instance, low-density, $n_{e}$ $\sim 10^{7} \mathrm{~cm}^{-3}$, non-neutral plasmas with temperature $T_{e}$ $\leqslant 1 \mathrm{keV}$ have been confined with static magnetic fields in Malmberg-Penning traps. ${ }^{1}$ Low-density, $n_{e} \sim 10^{14} \mathrm{~cm}^{-3}$, $T_{e} \sim 10-100 \mathrm{keV}$ plasmas are confined in magnetic mirrors and tokamaks. Since the discovery of the ponderomotive force over 40 years ago, it has been well known that charged particles interacting with an oscillating electromagnetic field will seek regions of the minimum light intensity (darkseeking behavior). ${ }^{2}$ The idea of trapping charged particles by the ponderomotive force with the appropriate electromagnetic field distribution was then proposed. ${ }^{3}$ Two-dimensional electron confinement with a specially shaped laser beam has been discussed. ${ }^{4-6}$ By modulating laser pulse intensities via wave-plates, a strong three-dimensional optical trap capable of confining electrons of kinetic energies up to $10 \mathrm{keV}$ was built. $^{7,8}$

In this paper, we discuss an optical trap capable of confining extremely high density (close to critical density) and hot (relativistic) plasmas, of kinetic energy up to $350 \mathrm{keV}$, by means of the interference of two terawatt-class (TW) femtosecond laser pulses. In the intersection region of laser beams, the modulated total laser intensity formed ponderomotive po-

\footnotetext{
a) Paper GI2 2, Bull. Am. Phys. Soc. 47, 137 (2002).

${ }^{b)}$ Invited speaker.

c) Also at Laboratory of Optical Physics, Chinese Academy of Sciences, Beijing 100080, People's Republic of China.
}

tential troughs of subwavelength width $(0.7 \mu \mathrm{m})$, and very high ponderomotive potential gradients, up to $10^{12} \mathrm{eV} / \mathrm{m}$. The Thomson scattering, stimulated Raman scattering, analysis, and computer simulation all indicate that the electrons were bunched by the strong ponderomotive force into sheets of thickness two orders of magnitude less than the laser wavelength, and an electron density up to 10 times higher than that of the background $n_{0}$. Correspondingly, the stimulated Raman side scattering indicates strong electron density deletion $\left(0.4 \%\right.$ of $\left.n_{0}\right)$ between the density-bunched regions. An electrostatic field of $10^{11} \mathrm{eV} / \mathrm{m}$ was produced by the bunched electrons. Unlike the electric field of an electron plasma wave, ${ }^{9-12}$ the electrostatic field in this optical trap was a localized direct-current field, with zero phase velocity and a fixed field direction during the laser beam interference.

\section{ANALYSIS AND COMPUTER SIMULATION}

The physical picture of this optical trap is simple. A ponderomotive force $\vec{F}_{p} \propto \nabla I$, where $I$ is the intensity of laser, is produced when light intensity has a spatial gradient. Two intense laser beams of the intensities $I_{1}$ and $I_{2}$, with same frequency and parallel polarization, perpendicularly crossing each other, interfere, causing spatial modulation of the light intensity given by $I=I_{1}+I_{2}+2 \sqrt{I_{1} I_{2}} \cos \delta$, where $\delta$ is the phase difference of these two laser pulses. In the experiment described in the following, $I_{1}=0.25 I_{2}$ and the peak interference intensity is nine times higher than that of the valley, and the distance of the intensity peak-to-valley is $0.35 \lambda_{L}$, where $\lambda_{L}$ is the wavelength of the laser. These intensity peaks and valleys lie along $x$, which is the spatial dimension perpendicular to the bisector of the two laser propagation directions. By means of the interference of two high-power laser pulses, a very high intensity gradient cre- 


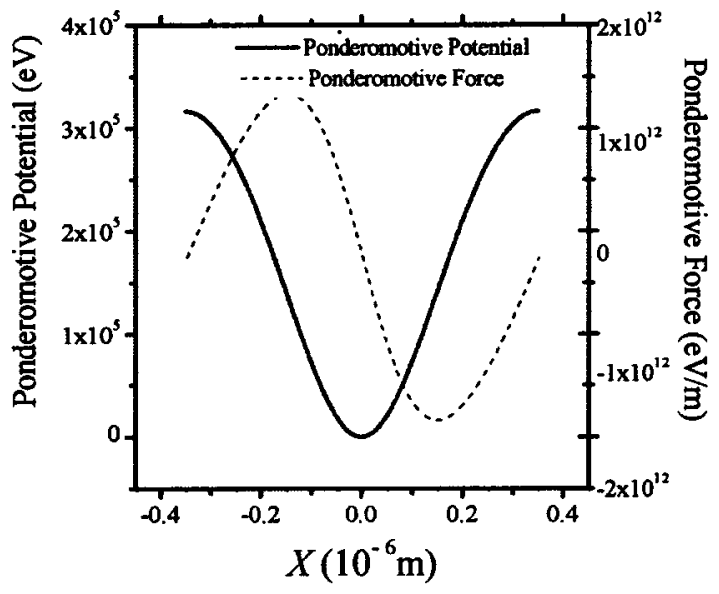

FIG. 1. Ponderomotive force and potential distribution. The potential valley forms the optical trap.

ated. If free electrons are present, they will oscillate in the high frequency laser field and Thomson-scatter light. On a time scale of several laser cycles, they experience a ponderomotive force that pushes them to the intensity valleys. The ponderomotive force is

$$
\vec{F}_{p}=-m_{e} c^{2} \frac{\partial \gamma}{\partial x},
$$

where $\gamma=\sqrt{1+a^{2} / 2}$ is the relativistic factor and $a=8.5$ $\times 10^{-10} \lambda_{L}(\mu \mathrm{m}) \sqrt{I\left(\mathrm{~W} / \mathrm{cm}^{2}\right)}$ is the normalized vector potential of the laser field, $\lambda_{L}$ is the laser wavelength, $I$ is the total intensity, and $m_{e} c^{2}$ is the rest-energy the electron. The interference laser intensity expressed by the normalized vector potential is $a^{2}=8 a_{1}^{2} \sin ^{2}(\pi x / D)$, where $D$ is the distance between the two laser intensity peaks and $|x| \leqslant D / 2$. The ponderomotive force can be calculated using Eq. (1), which gives

$$
\vec{F}_{p}=-\frac{2 \pi m_{e} c^{2} a_{1}^{2}}{\gamma D} \sin \left(\frac{2 \pi x}{D}\right) \frac{\vec{x}}{x} .
$$

Using the laser parameters in the experiment described in the following, in the interference region, the peak laser intensity is $4 \times 10^{18} \mathrm{~W} / \mathrm{cm}^{2}$. The corresponding ponderomotive force is up to $10^{12} \mathrm{eV} / \mathrm{m}$, and the ponderomotive potential $\phi_{p}$, defined by $\vec{F}_{p}=-\nabla \phi_{p}$ is about $300 \mathrm{keV}$ (Fig. 1).

Initially, because the plasma is uniform, the electrons experience only the ponderomotive force, which pushes them toward the interference troughs, where they are trapped and bunched. Because the much heavier ions do not have time to move significantly during the interference of subpicosecond duration pulses, but electrons do, a large directcurrent electrostatic field $\vec{E}_{\text {es }}$ is created, which exerts an electrostatic force on the electrons in the direction opposite to the ponderomotive force.

The value of electrostatic force $e \vec{E}_{\mathrm{es}}$ increases with bunching, based on Gauss' law

$$
\int_{S} \vec{E}_{\mathrm{es}} \cdot d \vec{S}=\frac{e}{\varepsilon_{0}} \int_{V}\left(n_{e}-n_{0}\right) d V,
$$

where $S$ and $V$ are the boundary surface and volume of the

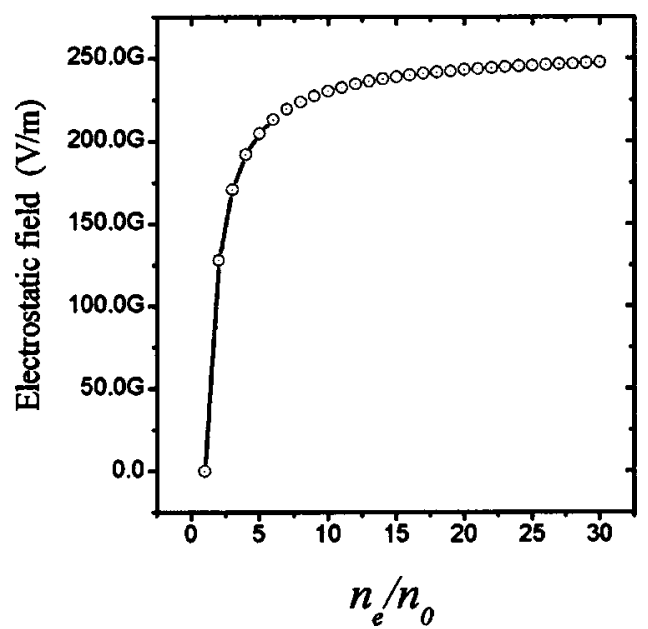

FIG. 2. Electrostatic field vs electron density ratio $n_{e} / n_{0}$.

bunched electrons, respectively, and $n_{e}$ and $n_{0}$ are the bunched and background electron (or positive charge) densities, respectively. This charge distribution is localized in the optical trap, but all these charged particles (inside the Gaussian pillbox) will contribute to the field no matter if these charges are in motion or not. When the bunched electron density $n_{e}$ is higher than $n_{0}$ in the pillbox during the laser beam interference, the direction of the electrostatic field is fixed and thus the field is a direct-current one. Assuming that the bunching process is in one dimension, the electrostatic force created by the electron bunch is given by

$$
\vec{E}=\frac{n_{0}|e|}{\varepsilon_{0}}\left(\frac{n_{e}}{n_{0}}-1\right) \vec{X}
$$

where $X$ is $1 / 2$ the thickness of the bunched electron sheet. The maximum intensity of the electrostatic field is reached at the boundaries of the pillbox. Using Eq. (3), the dependence of electrostatic field on the electron density ratio $n_{e} / n_{0}$ is shown in Fig. 2. In the experiment described in the following, the background electron density $n_{0}$ is $4 \times 10^{25} \mathrm{~m}^{-3}$. With $n_{e} / n_{0}=2$, the field strength jumps to $E_{\text {es }}=1.28$ $\times 10^{11} \mathrm{eV} / \mathrm{m}, \quad$ and when $n_{e} / n_{0}=6, \quad E_{\text {es }}$ reaches 2.1 $\times 10^{11} \mathrm{eV} / \mathrm{m}$, and then it increases gradually with $n_{e} / n_{0}$ to the saturation value of $\sim 2.5 \times 10^{11} \mathrm{eV} / \mathrm{m}$.

A similar grating-like electron distribution at the surface of a plasma was previously predicted and observed by means of a one-dimensional particle-in-cell code, ${ }^{23}$ but this model neglected the influence of electron thermal pressure.

In the bunch process, the force of electron thermal pressure $\vec{F}_{T}$ prevents the electron accumulation. Assuming that the bunch process is adiabatic, $\vec{F}_{T}$ and the electron thermal pressure $P_{e}$ are given by

$$
\begin{aligned}
& \vec{F}_{T}=\frac{\nabla P_{e}}{n_{e}}, \\
& P_{e}=n_{0} T_{e 0}(\mathrm{eV})\left(\frac{n_{e}}{n_{0}}\right)^{3}, \\
& n_{e}(x)=\alpha n_{0} \exp \left[\left(\frac{x}{X}\right)^{2}\right],
\end{aligned}
$$




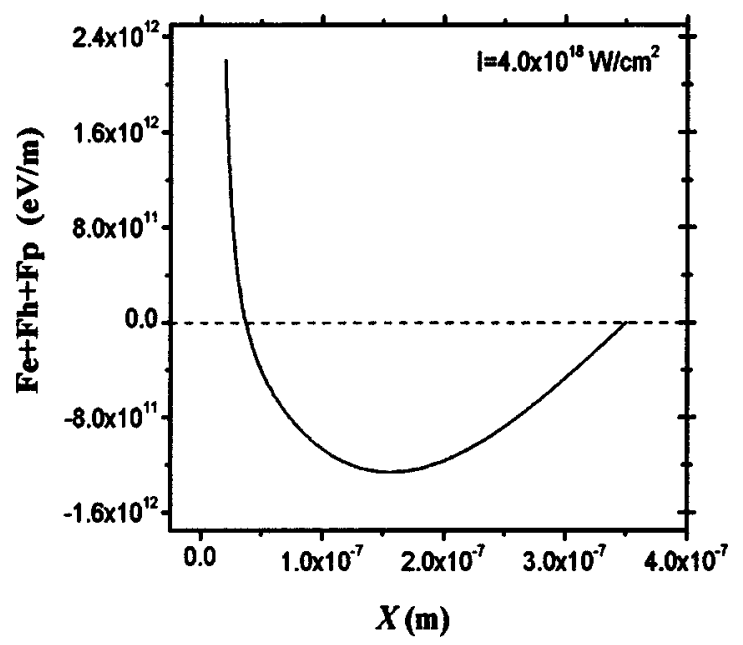

FIG. 3. Combination of the ponderomotive force, electrostatic force, and force of thermal pressure.

where $T_{e}(\mathrm{eV})=100 \mathrm{eV}$ is the initial electron temperature and $\alpha$ is a parameter determined by the restriction of electron number conservation

$$
\frac{\alpha}{D} \int_{-D / 2}^{D / 2} \exp \left[-\left(\frac{x}{X}\right)^{2}\right] d x=1 .
$$

Then the force of thermal pressure on the boundary of the bunched electron sheet is

$$
\vec{F}_{T}=6 \alpha^{2} T_{e 0}(e V) \frac{\vec{X}}{X^{2}} .
$$

Assuming the bunched electron sheet boundary locates at the force balance points where

$$
\vec{F}_{P}(X)+\vec{F}_{\text {es }}(X)+\vec{F}_{T}(X)=0,
$$

the thickness $(2 X)$ of the electron sheets and the corresponding electron densities at different interference intensities can be calculated. Figure 3 shows the dependence of the combination of the three forces on the thickness of the bunchedelectron sheets at peak laser intensity of $4 \times 10^{18} \mathrm{~W} / \mathrm{cm}^{2}$. The total force looks like van der Walls force, where at points beyond the balance point closer to the interference fringe, the force is a bunching force, while points closer to the bunched electron distribution, the force is thermalpressure dominated, resist the further accumulation. The electron density ratio $n_{e} / n_{0} \sim D / 2 X=9.2$ is then calculated. At peak laser intensity of $4.8 \times 10^{18} \mathrm{~W} / \mathrm{cm}^{2}$, the highest intensity observed in the experiment, the width of each bunched density region was then reduced to $0.68 \mu \mathrm{m}$ or about $D / 10.2$, which implies $n_{e} / n_{0}=10.2$ and $E_{\mathrm{es}} \sim 2.3$ $\times 10^{11} \mathrm{eV} / \mathrm{m}$.

The bunched electron in the laser beam intersection has the structure of a density grating or multi-layer mirror. It will diffract or reflect incident laser light resulting in laser energy transfer between the two crossed laser beams. Based on the density-grating model, the density grating satisfies

$$
\begin{aligned}
& D\left(\sin \theta_{m}-\sin \theta_{t}\right)=m \lambda, \\
& m=0, \pm 1, \pm 2, \ldots,
\end{aligned}
$$

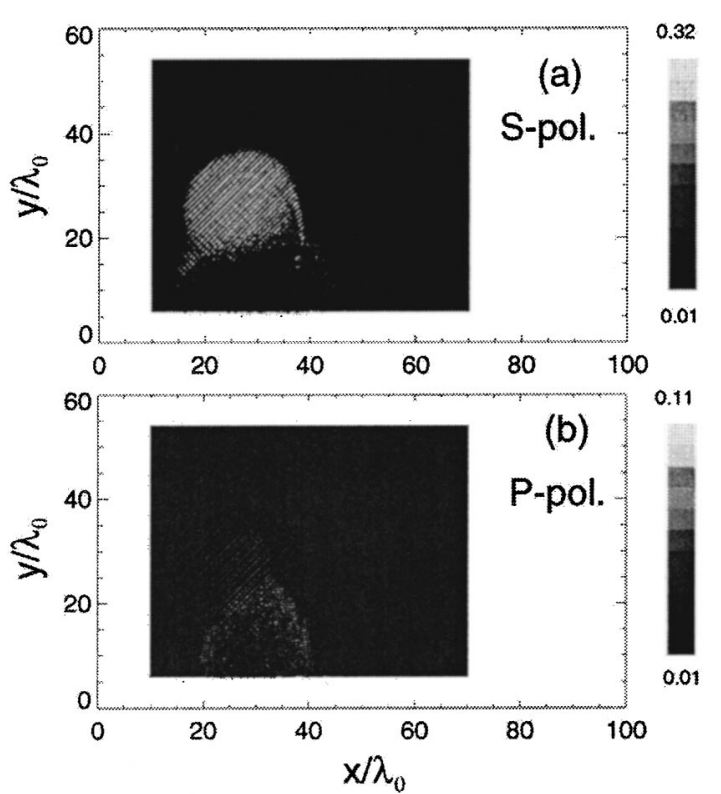

FIG. 4. Simulation shows that, with the interference of two $s$-polarized laser pulses, there is an electron-density bunching and grating structure at the laser intersection (upper picture), while, with two $p$-polarized laser pulses, no such density bunching (lower picture).

where $\theta_{i}$ is the incident angle and $\theta_{m}$ is the diffraction angle of $m$ order. In our experiment, the weaker laser beam $I_{1}$ was named pump and the stronger one $I_{2}$ was injection. If the injection beam is the incident laser, the only possible diffraction direction is in the pump direction with $(m=-1)$, and vice versa. The weaker pump beam will get more energy from injection during the dual beam interference. By using the multi-layer mirror mode, the same results of energy transfer are obtained.

The above-given calculation is consistent with a twodimensional particle-in-cell code computer simulation, which solves Maxwell's equations and the equation of motion for the particles in plasma. In this simulation, a rectangular simulation box of $100 \lambda \times 60 \lambda$ is used, which is split into $1000 \times 600$ cells for the integration of the Maxwell's equations. A homogeneous plasma volume with an initial density of $0.04 n_{c}$ occupies part of the simulation box. The pump laser of the normalized vector potential $a_{1}=0.5$, and it is along the $x$ direction. The injection pulse of $a_{2}=1.0$, which is four-times stronger in intensity than pump, is along the $y$ direction. Nine particles per cell are used for electrons and ions. Absorption boundaries for the fields and reflection boundaries for particles are used in both the $x$ and $y$ directions. The simulation results are depicted in Fig. 4, where the bunched charge regions exhibit a peak density ratio of $n_{e} / n_{0} \sim 10$ and width $\sim 1 / 10$ of the distance between interference peaks, at the time of maximum overlap. The simulation also predicts that the bunched electrons Thomson scatter the laser so that there is significant energy transfer from injection to pump, making the pump laser increase about $50 \%$ (Fig. 5). This energy transfer was also predicted by previous theory. ${ }^{13}$ 


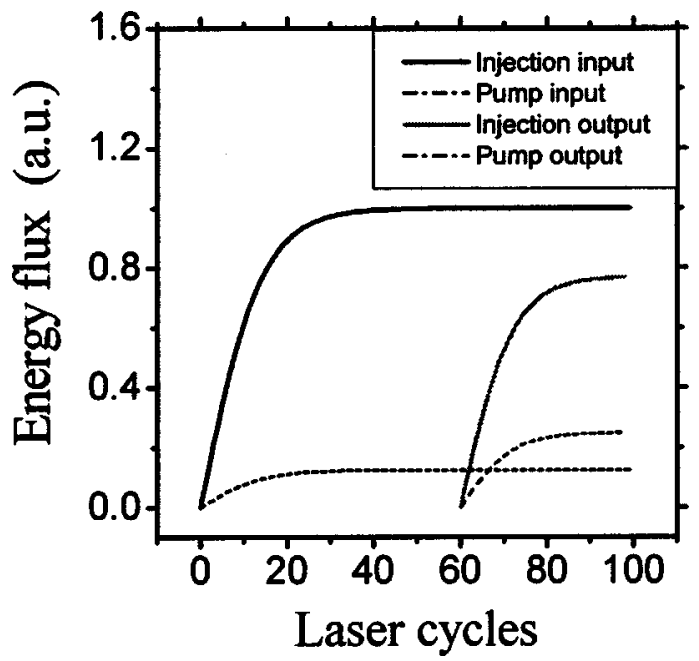

FIG. 5. Energy transfer from stronger injection laser to the weaker pump laser shown by simulation.

\section{EXPERIMENTAL RESULTS}

In a proof-of-principle experiment, two $1.053 \mu \mathrm{m}$ wavelength laser pulses, each ultra-short in duration (400 fs) and high peak power (1.5 and 6.0 TW), were focused perpendicularly to each other, with $f / 3$ parabolic (vacuum spot-size of $12 \mu \mathrm{m}$ full width at half maximum), reaching peak intensities of $6 \times 10^{17}$ and $2.4 \times 10^{18} \mathrm{~W} / \mathrm{cm}^{2}$, respectively. The beams were predominantly upward polarized, but had a small component of horizontal polarization due to the tight focusing geometry. Using a delay line, the pulses were overlapped temporally to within $30 \mathrm{fs}$ inside a supersonic helium gas jet (at $\left.5.5 \times 10^{6} \mathrm{~Pa}\right)$.

Plasma with density $n_{0}=4 \times 10^{25} \mathrm{~m}^{-3}$ was created by photoionization of the gas. Light propagation through the plasma was observed from top-view Thomson scattering pictures. The bright spot in Fig. 6 showed that the Thomson scattered light was significantly enhanced along the bisector of the laser beam intersection region. A line out of the bright spot indicated that the spatially averaged Thomson scattered power $\left\langle P_{s}\right\rangle$ from the region of the beam's intersection was more than ten times $\left\langle P_{0}\right\rangle$. The latter was from the background electrons outside the intersection region with density in the channel created by the more powerful of the two laser beams. This enhancement, $\left\langle P_{s} / P_{0}\right\rangle=10$, implied that the scattering was coherent, i.e., the Bragg scattering formula, ${ }^{14}$ $P_{s} / P_{0} \propto\left(n_{e} / n_{0}\right)^{2}$ applied, and indicated that $n_{e} / n_{0}>10$, which was 100 times higher than the largest reported amplitude for a plasma wave, which - unlike a trap modulationwas limited in amplitude by wave-breaking. ${ }^{15,16}$

The top-view spectra of the scattering lights are shown in Fig. 7. With only the pump laser, the signals of spectrum were in the level of background. When the two laser pulses were crossed, the spectrum clearly shows peaks of the stimulated Raman scattering (SRS) of the frequency shift $\Delta \omega$ $\sim \omega_{p}=1.9 \times 10^{13} \mathrm{arc} / \mathrm{sec}$ corresponding to plasma density $n_{e} \sim 4 \times 10^{-3} \quad n_{0}$ determined using $\omega_{p}=\sqrt{e^{2} n_{e} / \gamma \varepsilon_{0} m_{e}}$, where $\gamma$ was the relativistic factor and $\varepsilon_{0}=8.85$ $\times 10^{-12} \mathrm{~F} / \mathrm{m}$ was the permittivity of free space. The result
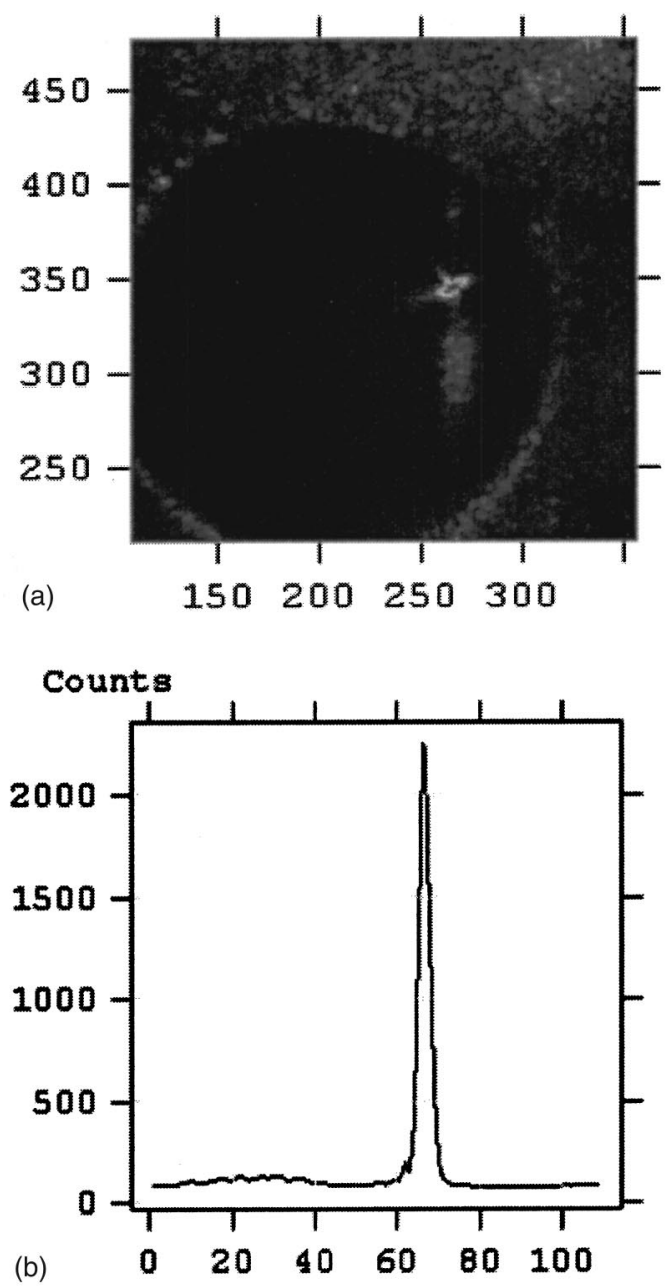

FIG. 6. (a) Image of the Thomson scattered light viewed from top downward to the throat of the nozzle. The weaker pump beam propagated from right to left while the stronger injection beam from top to bottom. (b) Intensity distribution of Thomson scattering light along the injection plasma channel showed the light enhancement at the beam intersection. From this result, an accumulated plasma density with amplitude $n_{e} \sim 10 n_{0}$ was inferred.

indicates relatively large density accumulation, about ten times of the background. Plasma cavities were dug to nearly $99.6 \%$ electron density depletion. Figure 7 also showed that the unshifted light, originating from Thomson scattering, was about five times stronger with crossed laser pulses than from only injection pulse. When the effects of spatial integration were accounted for, the ratio $\left\langle P_{s} / P_{0}\right\rangle \sim 10$ is again obtained.

With crossed laser pulses, two strong satellite lines were observed in the spectrum in Fig. 7, with the wavelength shifts $\sim \pm 3.8 \mathrm{~nm}$ away from the fundamental light. These two satellite lines may have originated from stimulated Brillouin scattering (SBS). The associated ion acoustic wave was excited by the beating or optical mixing of the crossed laser pulses, which had the frequency bandwidths that exceeded the ion acoustic frequency shift. The ion acoustic wave and SBS signals will be discussed in detail in a forthcoming publication.

The spectra of light scattered in the direction of pump beam were also measured (Fig. 8), and the results indicate 


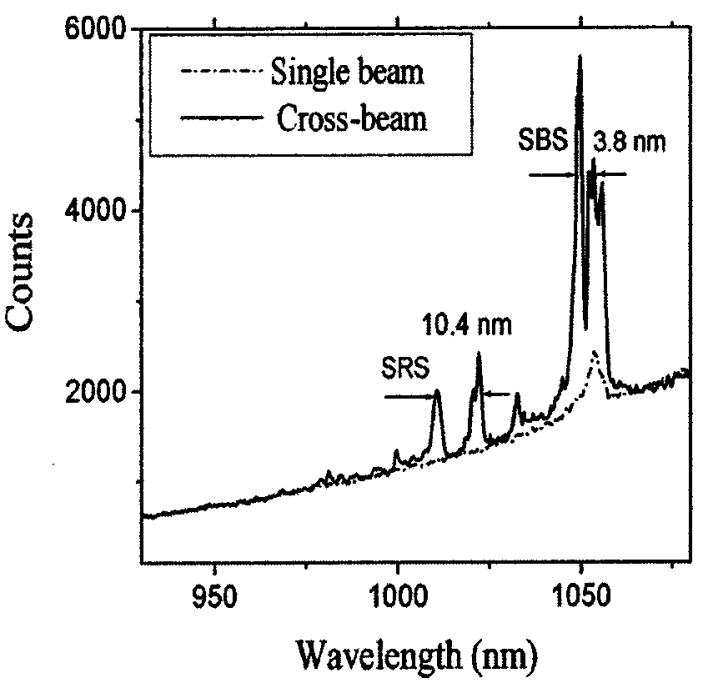

FIG. 7. Top view spectra of the scattered light, with/without the pump beam.

that the pump laser beam was enhanced by energy transfer. This result confirmed the prediction of the analysis, simulation, and theory. The bunched electrons not only reflected the fundamental laser but also all the scattering light signals from injection to pump or vice versa. The reflection of forward stimulated Raman scattering light from injection to pump may especially bring about optical mixing between the fundamental light and the reflected scattering, resulting in the pump plasma wave being resonantly driven. It was observed in some spectra that with dual pulse illumination, the scattered lights in the pump direction were obviously enhanced while the fundamental laser signal was barely increased. A possible reason is that resonant excitation of the plasma waves effectively absorbed the driving laser energy.

In order to test the calculation model prediction for the dependence of the $n_{e} / n_{0}$ on the laser interference intensities, the values of $\left\langle P_{s} / P_{0}\right\rangle$ were measured at peak laser intensities ranging from $8 \times 10^{17}$ to $4.8 \times 10^{18} \mathrm{~W} / \mathrm{cm}^{2}$. Discounting

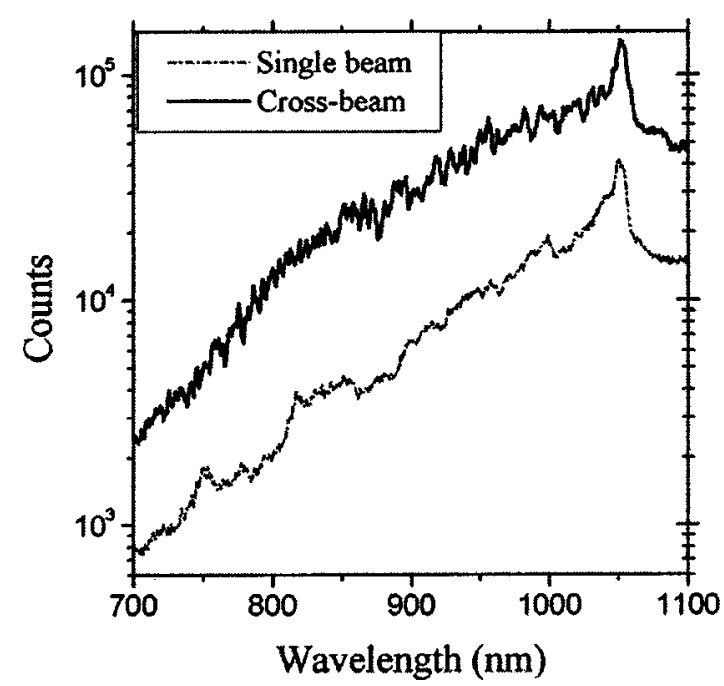

FIG. 8. Spectra of the laser and scattered light in the pump beam direction, with/without injection beam.

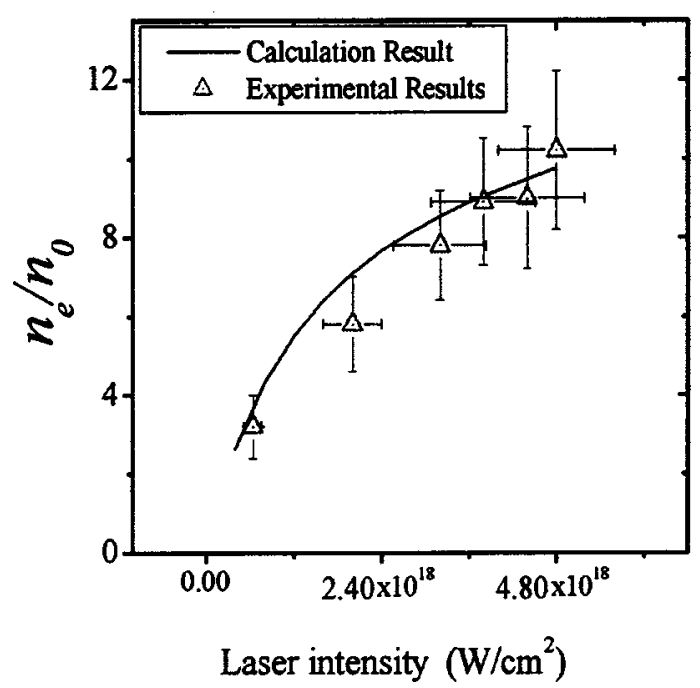

FIG. 9. Comparison of the analytical and experimental results of the inferred electron-density-ratio vs laser intensities.

the background and the contribution of SBS, the result was shown in Fig. 9, and the inferred values of $n_{e} / n_{0}$ coincided relatively well with the theoretical prediction.

\section{ELECTRON HEATING AND INJECTION}

We have previously discussed that the optical trap can bunch the electrons and produce high electron densities, up to $10 n_{0}$, resulting in the excitation of a strong electrostatic field, on the order of $10^{11} \mathrm{eV} / \mathrm{m}$. The resistance of the electrostatic force against the bunching ponderomotive force increases the electric potential energy of the bunched electrons. $P-V$ work will increase the electron temperature. Assuming that the bunching process is adiabatic, the temperature of the bunched electrons is

$$
T_{e}(\mathrm{eV})=\left(\frac{n_{e}}{n_{0}}\right)^{\Gamma-1} T_{e 0}(\mathrm{eV}) .
$$

If the process is in one dimension, $\Gamma=3$, with a ten-time electron density increase, the corresponding electron temperature $T_{e}(\mathrm{eV})$ will be increased by two orders.

It has to be pointed out that the above-mentioned adiabatic model works well in a quasi-static process in which Maxwell distribution applies. Actually in the short time period of the interference of $400 \mathrm{fs}$, the bunched electron system approaches, but never reaches, such an equilibrium state. The boundary of the accumulated electron bulk vibrates around the force balance points. If the vibration is assumed harmonic, the frequency of the vibration can be simply estimated from Fig. 3. Near the force balance point, the slope $\Delta F / \Delta X$ is about $-2.7 \mathrm{~N} / \mathrm{m}$, and thus a frequency 1.6 $\times 10^{15} \mathrm{~s}^{-15}$ can be calculated. The resistance of the electron thermal pressure against the bunching increases the electron kinetic energies of random motion, which is related to the electron temperature, and the work of electrostatic force increases the electron potential energies. With increases of the electron temperature and potential energy, the laser energy 


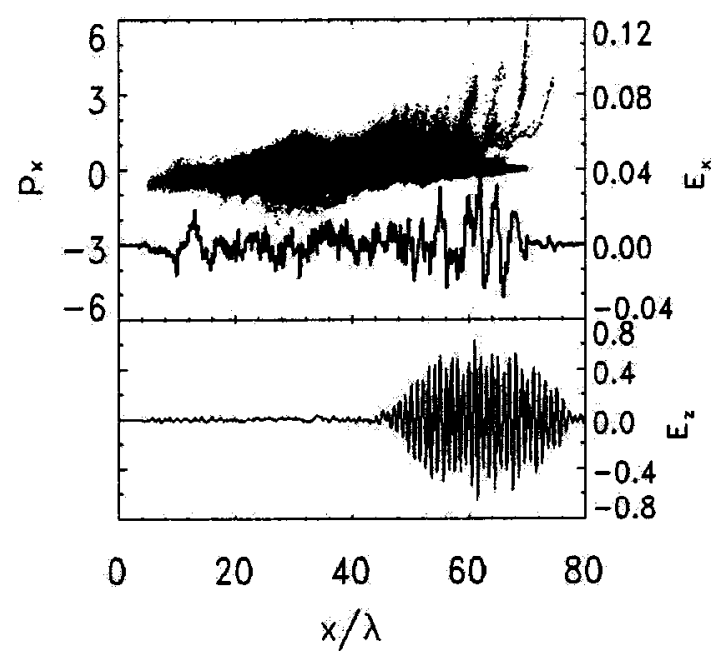

FIG. 10. Snapshot of simulation taken at 80 laser cycles shows that with dual laser illumination, the electric field in pump laser direction $x$ was obviously enhanced and electrons were heated and accelerated primarily in the pump-laser direction. The results were consistent with the electrons being heated during the period of beam overlap and then injected into the acceleration phase of the enhanced plasma waves in the pump direction.

is gradually absorbed by the bunched electrons and the vibration amplitude gradually decreases until the end of the laser pulse interference.

Computer simulation shows that the electrons are heated in the beam intersection and then these preheated electrons are injected into the enhanced pump plasma wave. Acceleration by the resulting plasma wave produces a beam of high energy electrons in the direction of pump (Fig. 10). The measurement of the electron spectra and beam profiles in pump direction with/without injection shows that with crossed laser pulses, the number of high energy electrons is increased three times and the corresponding temperature increase is more than $100 \mathrm{keV}$ (Fig. 11). Possible mechanisms for the stronger electron beam are the enhancement of pump plasma wave by the laser energy transfer, the beating of the reflected forward SRS light from injection with the pump laser light, and the injection of the preheated electrons into pump plasma wave, which made more electrons phase-matched with the wave. Simulations also indicated that by using this technique with shorter pulse lasers, the energy spread of these accelerators might be significantly reduced. ${ }^{17}$ Details of the effects of the two crossed laser pulses on electron acceleration in the laser driven plasma wave will be discussed in a separate publication.

\section{DIFFERENCES BETWEEN THE OPTICAL TRAP AND PLASMA WAVES}

The electrostatic field of our optical trap is different from that in a plasma wave (the field strength of a plasma wave can be on the order of $10^{11} \mathrm{eV} / \mathrm{m}$ ). First, the optical trap and the electrostatic field of the optical trap are localized and have zero phase velocity. A plasma wave, on the other hand, moves with velocity of $v_{p} \sim c \sqrt{1-\omega_{p}^{2} / \omega^{2}}$. Second, the distance (wavelength) between the two bunched electron density peaks is only $0.7 \lambda_{L}$, while in the plasma wave, the wavelength, based on the parameters of laser and back-
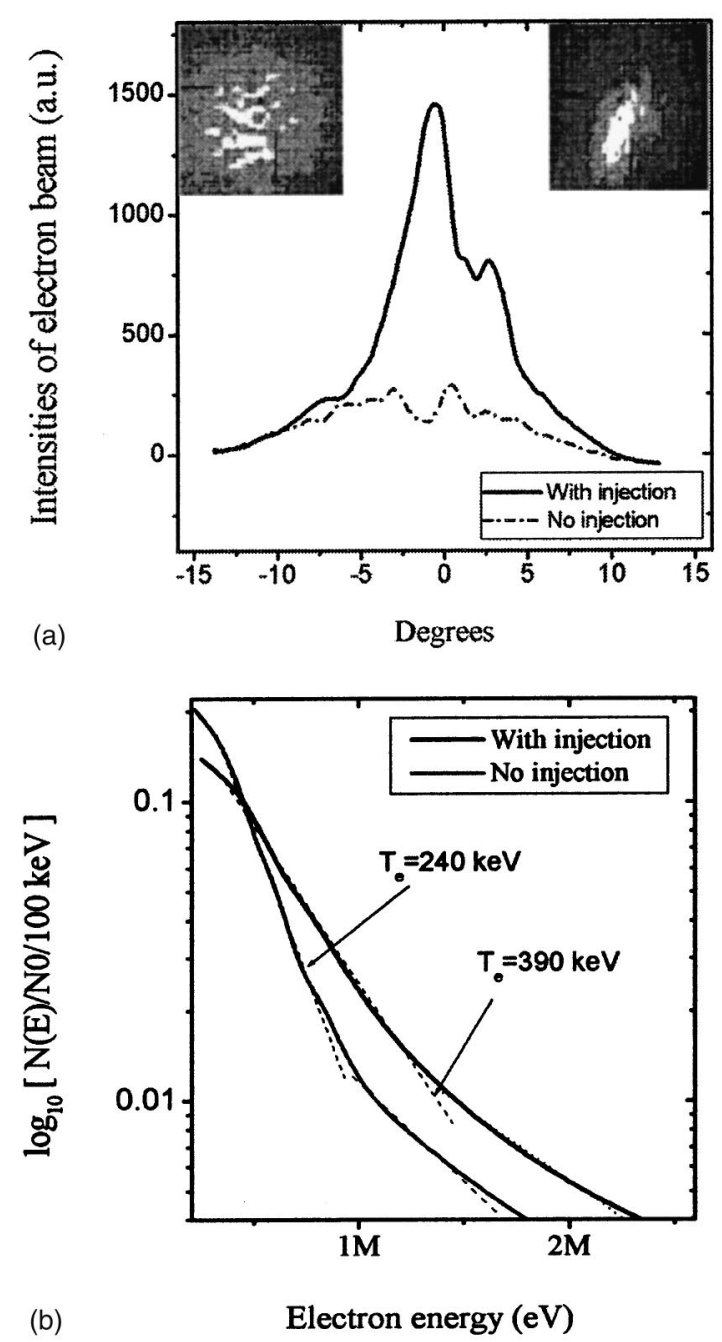

FIG. 11. (a) Enhancement of electron number with injection on upper left picture: the electron beam profile without injection. Upper right picture: the profile with injection. (b) Increase in electron temperature in pump beam direction with injection on.

ground plasma in our experiment, is much longer (by greater than ten times) than the laser wavelength. Third, the bunched electrons have density modulation $\delta n_{e} / n_{e}=\left(n_{e}-n_{0}\right) / n_{0}$ up to 10 , while in a plasma wave the corresponding density modulation is less than 1. Fourth, the function of the optical trap is to hold electrons and increase their electrostatic potentials and the kinetic energies of random motion. A plasma wave, on the other hand, acts to increase the electron kinetic energies of directional motion along the propagation direction. These differences make this optical trap unique in laser plasma physics.

There are several important applications of the optical trap besides electron acceleration. For instance, it might be used as a test bed for the study of relativistic nonlinear Thomson scattering. ${ }^{8}$ The ponderomotive force can be expressed in another form

$$
\vec{F}_{p}=-\nabla\left(T+m_{0} c^{2}\right)=-\nabla\left[m_{0} c^{2}\left(1+\frac{I_{18} \lambda_{L}^{2}}{1.37}\right)^{1 / 2}\right],
$$

where $T$ is the kinetic energy of the electrons and $I_{18}$ is the interference laser intensity in units of $10^{18} \mathrm{~W} / \mathrm{cm}^{2}$. If the 
interference intensity is $I_{18} \sim 10$, then $T_{\max }=1 \mathrm{MeV}$, or about $2 m_{e} c^{2}$. When the electrons oscillating with this energy collide with the stationary nuclei of plasma ions, they will produce positrons, ${ }^{18}$ which can either be accelerated in laser driven wakefield or allowed to annihilate with the electrons to generate bright gamma ray bursts with $511 \mathrm{keV}$ energy. This research is also relevant to fast ignition fusion ${ }^{19}$ or ion acceleration experiments, ${ }^{20}$ in which a laser pulse may potentially beat with a reflected weaker pulse, with intensities comparable to those used in our experiment. Last, an electron beam that enters the trap with kinetic energy exceeding the trapping threshold will be "wiggled" by the periodically spaced electrostatic field, causing emission of coherent shortwavelength radiation, as discussed previously in the context of plasma-wave wigglers. ${ }^{21}$ Remarkably, the strength of the optical-trap field is almost one million times higher, and the wavelength is almost a million times shorter, than a conventional magnetic wiggler. Calculations indicate that 100-times shorter wavelength light can be generated in the former case, with electrons of the same given energy.

\section{CONCLUSIONS}

By interfering two TW femtosecond laser pulses in plasma, an optical trap of potential depth $\sim 350 \mathrm{keV}$ was experimentally created. An unprecedented electron bunching of $n_{e} / n_{0} \sim 10$ was inferred from scattering diagnostics. A localized electrostatic field of strength $\sim 2 \times 10^{11} \mathrm{eV} / \mathrm{m}$ was excited by the electron accumulation inside the optical trap. Transfer of light energy from one beam to another was also observed. Optical mixing by two crossed laser pulses resonantly excited electron plasma waves and ion acoustic waves. As predicted by analysis and simulation, electrons were heated in the optical trap and these preheated electrons were then injected into the enhanced pump plasma wave, resulting in enhancements of the electron beam both in in- tensity and temperature. The latter is the first step toward the experimental realization of the laser injected laser acceleration concept (LILAC). ${ }^{22}$

\section{ACKNOWLEDGMENTS}

We thank A. Maksimchuk for help with the laser and useful comments from W. Theobald, G. Shvets, N. Fisch, and D. D. Meyerhofer.

We acknowledge support from the Department of Energy, Award No. DE-FG02-98ER41071 and the National Science Foundation Grants Nos. 0078581 and 0114336.

${ }^{1}$ F. M. Penning, Physica (Utrecht) 3, 873 (1936); J. H. Malmberg and J. S. de Grassie, Phys. Rev. Lett. 35, 517 (1975).

${ }^{2}$ H. A. H. Boot and R. B. R.-S. Harvie, Nature (London) 180, 1187 (1957).

${ }^{3}$ V. Gapanov and M. A. Miller, J. Exp. Theor. Phys. 34, 242 (1958).

${ }^{4}$ N. J. Phillips and J. J. Sanderson, Phys. Lett. 21, 533 (1966).

${ }^{5}$ U. Mohideen, H. W. K. Tom, R. R. Freeman et al., J. Opt. Soc. Am. B 9 , 2190 (1992).

${ }^{6}$ C. I. Moore, J. Mod. Opt. 39, 2171 (1992).

${ }^{7}$ J. L. Chaloupka, Y. Fisher, T. J. Kessler et al., Opt. Lett. 22, 1021 (1997).

${ }^{8}$ J. L. Chaloupka and D. D. Meyerhofer, Phys. Rev. Lett. 83, 4538 (1999).

${ }^{9}$ C. S. Liu and V. K. Tripathi, Interaction of Electromagnetic Waves With Electron Beams and Plasmas (World Scientific, Singapore, 1994).

${ }^{10}$ E. Esarey and P. Sprangle, IEEE Trans. Plasma Sci. 24, 252 (1996).

${ }^{11}$ D. Umstadter, S-Y. Chen, A. Maksimchuk et al., Science 273, 472 (1996).

${ }^{12}$ R. Wagner, S-Y. Chen, A. Maksimchuk, and D. Umstadter, Phys. Rev. Lett. 78, 3125 (1997).

${ }^{13}$ G. Shvets, N. J. Fisch, A. Pukhov et al., Phys. Rev. E 60, 2218 (1999).

${ }^{14}$ R. E. Slusher and C. M. Surko, Phys. Fluids 23, 472 (1980).

${ }^{15}$ J. M. Dawson, Phys. Rev. 133, 383 (1959).

${ }^{16}$ T. Tajima and J. M. Dawson, Phys. Rev. Lett. 43, 267 (1979).

${ }^{17}$ D. Umstadter, Phys. Plasmas 8, 1774 (2001), and references therein.

${ }^{18}$ E. P. Liang, S. C. Wilks, and M. Tabak, Phys. Rev. Lett. 81, 4887 (1998).

${ }^{19}$ M. Roth, T. E. Cowan, M. H. Key et al., Phys. Rev. Lett. 86, 436 (2001).

${ }^{20}$ Y. Sentoku, V. Y. Bychenkov, K. Flippo et al., Appl. Phys. B: Lasers Opt. 74, 207 (2002).

${ }^{21}$ R. L. Williams, C. E. Clayton, C. Joshi et al., IEEE Trans. Plasma Sci. 21, 156 (1993).

${ }^{22}$ D. Umstadter, J. K. Kim, and E. Dodd, Phys. Rev. Lett. 76, 2073 (1996).

${ }^{23}$ L. Plaja and L. Roso, Phys. Rev. E 56, 7142 (1997). 